\title{
The European Astrophysics Doctoral Network
}

\author{
J. Heyvaerts, Paris
}

(Université de Paris VII, Coordinator of E.A.D.N.)

Astrophysical research, both space and ground-based, is becoming more and more integrated at the European and sometimes global level. The cost and technical complexity of the ambitious instruments that are needed to reach the frontiers of observational discovery leave no room for an alternative.

A group of University professors, heading important doctoral formations in their respective countries, felt that it was time to open to Ph.D. students also all the possibilities offered by European research in our field, and gathered to form a "European Astrophysics Doctoral Network". The aim of the Network is to stimulate the mobility of students preparing their doctoral theses, in both national and multinational institutions.

The importance of such mobility at the start of a student's first significant scientific work stems from the fact that contact with a wide community improves considerably the quality of the research and the insight of the student. Whilst the American community has benefited from such advantages for some time, little has been done up till now to offer the same opportunity to European research students. At the same time, the increasing multinational basis of much of European research calls for a more systematic circulation of young research workers, even at the predoctoral level.

\section{The European Astrophysics Doctoral Network}

At the start of the project, it was felt that for easy management, the network should be limited to one or at most two universities per country. It now associates 13 universities in 11 countries as seen in the panel. ESA and the ESO have their representative, and IRAM will soon join. We expect also participation from a Danish university and from a Swedish university. The Network has now been operating for three years, informally and on an experimental basis at the start, progressively evolving towards more formal arrangements.

It proposes organizing, and financing, a doctoral exchange programme, by offering to a small number of very good students the possibility of preparing their theses entirely abroad, and organizing opportunities for others, for shorter visits during the period of their doctoral studies, in appropriate foreign laboratories. The Network also proposes organizing European Summer Schools designed especially for doctoral students, the lectures being given by prominent experts at a high level. It also wants to support exchanges of graduates and undergraduates, as well as professors in the fields of astronomy, astrophysics and space sciences.

The Network receives funds for developing its activites from the member universities and various institutions in their respective states. It also applies for funding under programmes of the EEC, like JSP or ERASMUS, and seeks to participate in actions taken by the European Council. Since our objective is to make students and their teachers think in terms of Europe as an entity and to forget about borders, the concept of a genuinely collective European funding is much more appealing than gathering national contributions, as the money then carries no flag. It is also necessary for the project to proceed.

\section{Student Mobility Programme}

Over two years or so, we have been able to fund 38 visits of Ph.D. students abroad lasting between two or three months and one year each.

We want to develop this activity a lot more. The potential for doing so exists. Not only are colleagues very willing to receive a visiting student, but the feeling is also spreading among students that this is a very useful experience. In France, where we had been somewhat better funded at the start, some 14 such actions could be organized in two years. This means that if a similar rate of mobility would have been possible over all countries participating in the Network, a flux of some 70 to 80 visiting students would have been easily reached in this same time span.

There are some difficulties, however. One is the reluctance of students to
Present Members of the EADN

Austria: Universität Wien (Pr. Maitzen)

Belgium: Vrije Universiteit Brussel (Pr. de Loore)

Université de Liège (Dr Swings)

F.R. Germany: Universität Heidelberg (Pr. Appenzeller)

Spain:Universidad de la Laguna (Tenerife) (Pr. Rocca Cortez)

France: Université de Paris VII (Pr. Heyvaerts)

Greece: Panepistimio Athenon (Pr. Contopoulos)

Panepistimio Kritis (Pr. Ventura)

Italy: Università di Firenze (Pr. Chiuderi)

The Netherlands: Rijksuniversiteit te Leiden (Pr. Habing)

Portugal: Universidade do Porto (Pr. Lago)

Switzerland: Université de Genève (Pr. Maeder)

United Kingdom: University of Edinburgh (Pr. Longair)

move: this is slowly evolving in the right direction. Differences in regulations and level of tuition fees in various universities also occasionally cause difficulties.

The most worrying thing however, is the administrative complication and stiffness in the management of the ambitious CEE programme. In the "experimental" period $(86 / 87 / 88)$ which we enjoyed very much, the funds provided by CEE were handled in a flexible way. Actions could then be decided and realized almost "in real time". The board of the Network kept in touch, by meeting or by mail, quasi permanently, so that sometimes the delay between an application, the decision, and the ensuing action was not more than a couple of weeks. When moving to the more ambitious ERASMUS programme, it was felt that national agencies should administrate their share of the exchange grants programme. A rather detailed description of planned mobility actions, specifying how many students will go from this country to that other must be given in the proposal, which is due almost one year in advance. This means in practice that all these mobility actions have now to be arranged one year or more in advance. This is definitely not the appropriate time scale to manage mobility actions of Ph.D. students!

\section{European Predoctoral School of Astro- physics}

The first of our European Schools was organized in 1988, by P. Lena (Paris), $\mathrm{H}$. Habing (Leiden) and I. Appenzeller (Heidelberg) as a cooperation between the Network and the Fédération Française des Magistères de Physique. It took 
place in "Les Houches" from 5-16 September. We offered two topics:

- origin, structure and evolution of galaxies,

- methods and tools of astronomical observation.

The first was more fundamental, and the second more methodological. We plan to retain this scheme for our future schools, since it gives to each student both a major and a minor theme of interest, and keeps the scope of the teaching broad enough.

The School resources came 26\% from European funds (EEC programme, and European Council) and $74 \%$ from national contributions raised by Network members. All contributions were included in a global budget regardless of their origin. Good financial conditions were offered by the Board (Conseil d'Administration) of Les Houches "Ecole de Physique", in view of the wish of this Board to develop a pre-doctoral school.

Since the spirit of the School was to foster interaction, lecturers were asked to be present as much as possible during the School. Four stayed 2 weeks, four 1 week, one half a week. Springer Verlag (Heidelberg) are publishing a book based on the School material designed to become a series. The $1989 \mathrm{School}$ is planned be held in Ponte de Lima (Portugal) from 11-24 September 1989. The topics will be late stages of stellar evolution and numerical hydrodynamics in astrophysics.

\section{Physical Mechanisms in Polymer Failure}

\section{H.H. Kausch and W.J. Cantwell, Lausanne}

\author{
Report on the 20th Europhysics Conference on Macromolecular Physics/ \\ 3rd Lausanne Polymer Meeting, 26-30 September 1988, Lausanne.
}

For the first time in its 16 years of existence, the Europhysics Conference on Macromolecular Physics was held in Switzerland and following the tradition of the Lausanne Polymer Laboratory "Physical Mechanisms in Polymer Failure" was chosen as the conference theme. This topic was viewed from all possible levels and directions, ranging from the molecular scale of small deformations to the macroscopic behaviour of fibre-reinforced composites.

The general theme was subdivided into seven topics:

- Molecular Mechanisms and Theory of Deformation of Polymers

- Micromechanics of Crazing

- Crazing and Fracture

- Fracture and Degradation

- Highly Oriented Fibres

- Polymer Blends

- Fracture of Composites.

Within these subject areas, 30 invited lectures and ensuing discussions covered major recent developments.

\section{Physics of Macromolecules}

The powerful tool of neutron scattering was detailed by $M$. Dettenmaier (Mainz) who showed that the deformation of a molecule (of polycarbonate) is an affine image of the macroscopic deformation at temperatures up to $120^{\circ} \mathrm{C}$. The large molecular anisotropy, there- fore, plays a specific role only close to the glass transition temperature $T_{\mathrm{g}}$ (at $140^{\circ} \mathrm{C}$ ). B. Jasse (Paris) continued this theme and reported an unexpected finding: the first step in the plastic elongation of a sample at the yield point is a slight shortening of the chains by an increase in the number of gauche bonds - interpreted as being due to a shift in thermal configurations equilibrium.

As opposed to the previous discrete molecular approaches, C. G'Sell (Nancy) derived continuum mechanical constitutive equations, linked however, to molecular interpretations. Noteworthy, was the definition of micro-shear domains enlarged by plastic cycling and which favour local molecular mobility. Such a model permits the quantitative description of "rejuvenation" and "physical aging" phenomena.

The above considerations concern molecular segments; they are generally restricted to the range of linear viscoelastic behaviour. All strength properties depend, however, on the nature of the interaction of whole chains, of their interdiffusion and entanglement. $M$. Tirrell (Minneapolis) treated the materials science aspects of these mechanisms (see his extensive report given in $A n n$. Rev. Mat. Sci. 19, 1988).

One of the most intriguing mechanical phenomena in glassy polymers is the formation of crazes. This subject was treated in detail at the meeting and real breakthroughs were witnessed in the determination and interpretation of the fibrillation process introducing such new concepts as forced reptation (E.J. Kramer, Ithaca) and C.J. Plummer (Cambridge/UK), and stress induced sorption of otherwise non-compatible polymers (R.E. Cohen, Cambridge/Mass.). Most of the outstanding lectures of this day are published in a new volume of Advances in Polymer Science, Crazing in Polymers, Vol. 2, ed. H.H. Kausch, 1989). Another important subject is the fracture of polymers at low temperatures. As G. Gartwig (Karlsruhe) pointed out, at low temperatures the strength of a polymer increases whilst the fracture strain decreases. Most mechanical and thermal properties at very low temperatures are rather independent of chemical structure but depend on morphology, i.e. crystallinity or cross-linking. Unexpected, certainly, was that some polymers (PE) at $77 \mathrm{~K}$ become remarkably tougher at strain rates above $\dot{\varepsilon}=10^{3} \mathrm{~s}^{-1}$. On the third day of the conference, fracture, molecular structure and morphology were put into perspective. L.C.E. Struick (Geleen) chose the fracture mechanics approach and U.W. Gedde (Stockholm) a detailed analysis of the crystalline superstructure (including DSC and electron microscopy).

\section{Chemistry of Fracture}

Molecular fracture events were studied by F. Szöcs (Bratislava) using ESRtechnique; N.Ya. Rapoport (Moskow) drew attention to the generally neglected phenomenon that mechanical stresses can influence the kinetics of chemical reactions (e.g. the oxidation, leading to microcrack formation and subsequent, stress-dependent failure). She also pointed out the anisotropy of diffusion of low molecular weight radicals in stretched polymers - and thus the anisotropy of the propagation of the reaction.

In an attempt to elucidate the importance of the different molecular fracture mechanisms and exploiting the possibilities of a large chemistry laboratory, an exploring physicist, M. Fischer (Marly) had prepared networks with tailored distances, $L$, between crosslinking points. The critical crack opening in these materials turned out to be a constant multiple $(\cong 350$ ) of $L$; the fracture energy, however, was predominantly spent in the plastic deformation of the network up to such an extension.

J.T. Dickinson (Pullmann, Washington) discussed the application of fracto- 